\title{
A facility coloring problem in $1-\mathrm{D}^{\star}$
}

\author{
Sandip Das ${ }^{1}$, Anil Maheshwari ${ }^{2}$, Ayan Nandy ${ }^{1}$ and Michiel Smid ${ }^{2}$ \\ ${ }^{1}$ Indian Statistical Institute, Kolkata 700 108, India \\ ${ }^{2}$ School of Computer Science, Carleton University, Ottawa, Canada
}

\begin{abstract}
Consider a line segment $R$ consisting of $n$ facilities. Each facility is a point on $R$ and it needs to be assigned exactly one of the colors from a given palette of $c$ colors. At an instant of time only the facilities of one particular color are 'active' and all other facilities are 'dormant'. For the set of facilities of a particular color, we compute the one dimensional Voronoi diagram, and find the cell, i.e, a segment of maximum length. The users are assumed to be uniformly distributed over $R$ and they travel to the nearest among the facilities of that particular color that is active. Our objective is to assign colors to the facilities in such a way that the length of the longest cell is minimized. We solve this optimization problem for various values of $n$ and $c$. We propose an optimal coloring scheme for the number of facilities $n$ being a multiple of $c$ as well as for the general case where $n$ is not a multiple of $c$. When $\mathrm{n}$ is a multiple of $c$, we compute an optimal scheme in $\Theta(n)$ time. For the general case, we propose a coloring scheme that returns the optimal in $O\left(n^{2} \log n\right)$ time.
\end{abstract}

\section{Introduction}

In this paper we study a facility location problem. There are $n$ facilities to be distributed between $c$ classes of service providers. Each class of service provider should be assigned at least one facility and no facility should be assigned to more than one class. Moreover, when one class of service provider is active, all other classes are dormant. Our objective is to partition the set of facilities in $c$ classes such that the users are served as equitably as possible, i.e., the maximum length amongst the regions covered by any facility of any class is minimized.

In the area of wireless sensor networks, an effective approach for energy conservation is scheduling sleep intervals for sensors [1]. One can assign a color to each sensor, each color representing a set of sensors which would be active at a given time when the rest are in the sleep mode. Here the objective would be to color the nodes such that the maximum area covered by any active node is minimized. Lin et al [2] have explored the problem of maximizing the lifetime of wireless sensor networks. Their study is based on finding the maximum number of disjoint connected covers that satisfy both sensing coverage and network connectivity.

\footnotetext{
* This research is partially supported by NSERC and the Commonwealth Scholarship Program of DFAIT, Canada.
} 
Here we study the MinVor problem. Let $n_{\eta}$ be the number of facilities of color class $\eta$, where $\eta=0, \ldots, c-1$ and $n=\Sigma_{\eta=0}^{c-1} n_{\eta}$. For each $\eta$, we draw the Voronoi diagram considering the corresponding $n_{\eta}$ facilities which represents the active sensors while the remaining $n-n_{\eta}$ facilities represent the sensors in sleep mode. A Voronoi diagram of a set of $k$ sites partitions the Euclidean space into $k$ regions such that the region of each site consists of all points that are closer to it than to any other site. For our problem, the region $R$ is a horizontal line segment. Let $\gamma_{\eta, j}$ denote the length of the Voronoi zones of the $j$-th facility from the left end of some color $\eta$.

Formally, the MinVor problem is to devise a coloring scheme that minimizes $\max _{\eta=0,1, \ldots, c-1} \max _{j=1,2, \ldots, j_{\eta}} \gamma_{\eta, j}$ where $j_{\eta}$ is the total number of facilities assigned the color $\eta$.

Problems similar to the MinVor problem in the plane have been considered in $[1,3-5]$. Funke et al [1] presented a greedy algorithm that provides complete coverage with an approximation factor no better than $\Omega(\log n)$, where $n$ is the number of sensor nodes. An algorithm is said to provide complete coverage if the set of the selected sensors always covers the region $R$, provided that there exists a feasible solution. The communication graph is an undirected graph in which sensors are represented as nodes and there is an edge between two nodes if they can talk to each other. Attempts have been made to cover the Communication Graph using a connected dominating set (CDS) $S^{\prime}$, which is a subset of the set of sensors, $S$, such that each node in $S \backslash S^{\prime}$ is adjacent to some node in $S^{\prime}$ and the communication subgraph induced by $S^{\prime}$ is connected. Clark et al [3] have shown that the problem of finding a minimum CDS for unit-disk graphs is NPhard. An 8-approximation algorithm with $O(n)$ time complexity was suggested by Wan et al [4] which was later improved to a 6.91 approximation factor [5].

In this paper we consider the case where $R$ is a horizontal line segment and provide optimal solutions. As input to the problem we have the location of $n$ facilities, specified by a distance vector $\bar{d}=\left\langle d_{1}, d_{2}, \ldots, d_{n+1}\right\rangle$, where the $i$-th facility is placed at a distance of $\sum_{j=1}^{i} d_{j}$ from the left end of $R$, for $i=1, \ldots, n$ and $d_{n+1}$ is the distance of the $n$-th facility from the right end of $R$. We consider various cases depending on the values of $n$ and $c$. Note that each facility is assigned exactly one of the colors from $\{0,1, \ldots, c-1\}$ and each color is assigned to at least one facility. We assume that the density of users is uniform over $R$ and hence the Voronoi length reflects the proportional user volume.

For $n<2 c$ observe that there has to be a color with only one facility whose Voronoi zone is the whole space. In Section 3 we prove that $C_{1}$ (see Definition 1) is an optimal coloring for any distance vector if the number of facilities is twice the number of colors. In Section 4 we show that if the number of facilities is any multiple of $c$, the same coloring scheme provides an optimal solution. Section 5 suggests a coloring scheme for the general case where $n$ is not a multiple of $c$ which produces an optimal coloring in $O\left(n^{2} \log n\right)$ time. 


\section{Notations and definitions}

The facilities on the horizontal line segment $R$ are to be assigned colors from the set $\{0,1, \ldots, c-1\}$. A facility at position $f$ means that among the $n$ facilities on the line segment $R$, it is the $f$-th one from the left and hence at a distance $\Sigma_{k=1}^{f} d_{k}$ from the left boundary of the line segment $R . P_{i, j}$ is the position of the $j$-th facility from the left belonging to the color class $i$, i.e., there are exactly $P_{i, j}-1$ facilities to the left of this facility among which $j-1$ are of color $i$. We define $M(a, b)=\frac{1}{2} \sum_{\ell=a+1}^{b} d_{\ell}$, where $M(a, b)$ is the Voronoi length of a facility at some position $f$ whose immediate left neighbor of the same color is at position $a$ and whose immediate right neighbor of the same color is at position $b, a<f<b$ (see Figure 1 for an illustration). We define $L(a, b)=\Sigma_{\ell=1}^{a} d_{\ell}+\frac{1}{2} \Sigma_{\ell=a+1}^{b} d_{\ell}$, where $L(a, b)$ is the Voronoi length of a facility at position $a$ which is the leftmost of its color and whose immediate right neighbor (of the same color) is at position $b$. Analogously, we define $R(a, b)=\frac{1}{2} \Sigma_{\ell=a+1}^{b} d_{\ell}+\sum_{\ell=b+1}^{n+1} d_{\ell}$, where $R(a, b)$ is the Voronoi length of a facility at position $b$ which is the rightmost of its color and whose immediate left neighbor (of the same color) is at position $a$.

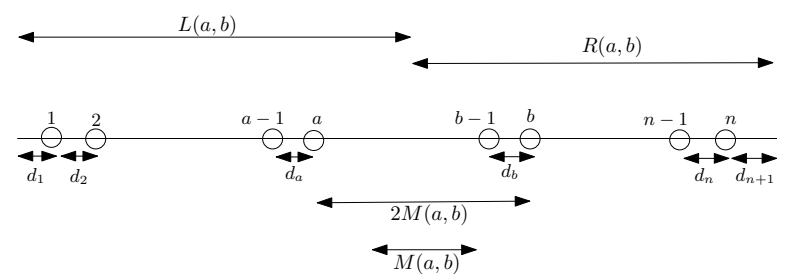

Fig. 1. $M(a, b), L(a, b)$ and $R(a, b)$

We define the objective function of a coloring $\mathrm{C}$ as $\Delta(\mathrm{C})$, which is the largest Voronoi length among all the facilities corresponding to all the colors.

In this paper we use certain fixed coloring schemes, as they turn out to be optimal for specific configurations. One such scheme is as follows.

Definition 1. (Coloring $\mathrm{C}_{1}$ ): Consider a coloring of the facilities from the left in the order $0,1, \ldots, c-1, c-1, \ldots, 0,0,1, \ldots, c-1, c-1, \ldots, 0, \ldots$ We define this assignment of colors as the coloring $\mathrm{C}_{1}$. The position of the $j$ th facility of color $i$ for $\mathrm{C}_{1}$ is denoted by $F_{i, j}$. Note that $F_{i, j}=2 c\left\lfloor\frac{j-1}{2}\right\rfloor+(2 c-i)\left(1-\chi_{j}\right)+(i+1) \chi_{j}$, where $\chi_{j}=1$ if $j$ is odd and 0 otherwise.

For example, for $n=9$ and $c=3, C_{1}$ will color the facilities by the colors $0,1,2,2,1,0,0,1,2$, from left to right on $R$ (see Figure 2). Let $\alpha_{i, j}$ be the length of the Voronoi cell of the $j$-th facility from the left belonging to the color class $i$, where facilities are colored by $\mathrm{C}_{1}$. Note that

$$
\alpha_{i, j}= \begin{cases}M\left(F_{i, j-1}, F_{i, j+1}\right) & \text { if } j \neq 1, j_{i} \\ L\left(F_{i, j}, F_{i, j+1}\right) & \text { if } j=1 \\ R\left(F_{i, j-1}, F_{i, j}\right) & \text { if } j=j_{i}\end{cases}
$$




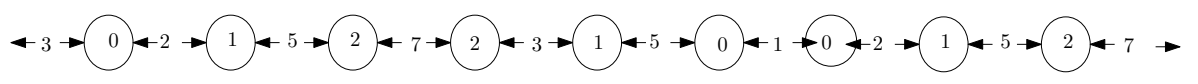

Fig. 2. An example for $n=9, c=3$ using $C_{1}$. The facilities are represented by circles and the numbers 0,1 or 2 within the facility represent the color of the facility. The distance vector is $\bar{d}=\langle 3,2,5,7,3,5,1,2,5,7\rangle$. The length of Voronoi cells of the facilities are as follows: $\alpha_{0,1}=L(1,6)=3+\frac{(2+5+7+3+5)}{2}=14$, $\alpha_{0,2}=M(1,7)=\frac{(2+5+7+3+5+1)}{2}=11.5, \alpha_{0,3}=R(6,7)=\frac{1}{2}+(2+5+7)=14.5$, $\alpha_{1,1}=L(2,5)=(3+2)+\frac{(5+7+3)}{2}=12.5, \alpha_{1,2}=M(2,8)=\frac{(5+7+3+5+1+2)}{2}=11.5$, $\alpha_{1,3}=R(5,8)=\frac{(5+1+2)}{2}+(5+7)=16, \alpha_{2,1}=L(3,4)=(3+2+5)+\frac{7}{2}=13.5$, $\alpha_{2,2}=M(3,9)=\frac{(7+3+5+1+2+5)}{2}=11.5, \alpha_{2,3}=R(4,9)=\frac{(3+5+1+2+5)}{2}+7=15$. Therefore $\Delta\left(\mathrm{C}_{1}\right)=\alpha_{1,3}=16$.

$\Delta\left(\mathrm{C}_{1}\right)=\max \left\{\alpha_{i, j}\right\}$, for all $i=0, \ldots, c-1$ and $j=1,2, \ldots, j_{i}$, where $j_{i}$ is the total number of facilities assigned to color $i$.

The value of the objective function for the optimum coloring is denoted by Opt.

\section{$3 n=2 c$}

In Theorem 1 we show that $C_{1}$ is an optimal coloring when we have $n=2 c$ facilities. It is obvious that for each color there will be exactly two facilities, otherwise the Voronoi cell for at least one of the colors will be whole of $R$, which is clearly non-optimal. In Case 1 of Theorem 1 , we show that if the objective function for $C_{1}$ returns the Voronoi length of the 1 st facility of some color $i$ (i.e. $\left.\Delta\left(C_{1}\right)=\alpha_{i, 1}\right)$, then any attempt to get a new coloring to reduce $\alpha_{i, 1}$ will ensure that in the new coloring there will be some color $i^{\prime}$, such that the length of the Voronoi cell corresponding to its 1 st facility will be at least $\alpha_{i, 1}$. The analogous result for the case where the objective function for $C_{1}$ returns the Voronoi length of 2 nd facility of some color $i$ is shown in Case 2 of Theorem 1. Using these two cases, we show in Theorem 1 that for $n=2 c, \mathrm{C}_{1}$ is an optimal coloring.

We consider $C^{\prime}$ to be a coloring scheme different from $C_{1}$ introduced as a candidate for possible improvement over $\mathrm{C}_{1}$. Let $\alpha_{i, j}^{\prime}$ denote the length of the Voronoi cell corresponding to the $j$-th facility from the left belonging to the color class $i$ in $C^{\prime}$. Let $F_{i, j}^{\prime}$ be the position of the $j$-th facility from the left of color $i$ using $C^{\prime}$.

Theorem 1. For $n=2 c, \mathrm{C}_{1}$ is an optimal coloring for the MinVor problem.

Proof. Suppose $\Delta\left(\mathrm{C}_{1}\right)=\alpha_{i, \ell}$ for some $i \in\{0, \ldots, c-1\}$ and some $\ell \in\{1,2\}$ for $\mathrm{C}_{1}$. 
Case 1: $\ell=1$ : We investigate if it is possible to achieve an objective function whose value is smaller than $\Delta\left(C_{1}\right)$ by any alternate coloring scheme $C^{\prime} . F_{j, k}^{\prime}$ is the position of the $k$-th facility of color $j$ for the coloring $\mathrm{C}^{\prime}$ and $\alpha_{j, k}^{\prime}$ be the Voronoi length of the facility at $F_{j, k}^{\prime}$ in $C^{\prime}$. Note that each color in $C^{\prime}$ is assigned to exactly two facilities. Let $S_{1}=\left\{i^{\prime}: F_{i^{\prime}, 1}^{\prime} \geq i+1\right\}$, i.e., $S_{1}$ is the set of colors whose first facility for coloring $\mathrm{C}^{\prime}$ is at position $i+1$ or higher. Clearly $\left|S_{1}\right| \geq c-i$. Note $F_{i^{\prime}, 1}^{\prime} \geq F_{i, 2}$ implies $\alpha_{i^{\prime}, 1}^{\prime}=L\left(F_{i^{\prime}, 1}^{\prime}, F_{i^{\prime}, 2}^{\prime}\right) \geq L\left(F_{i, 1}, F_{i, 2}\right) \geq \alpha_{i, 1}=\Delta\left(C_{1}\right)$. Hence $F_{i^{\prime}, 1}^{\prime}<F_{i, 2}=2 c-i \forall i^{\prime} \in\{0, \ldots, c-1\}$ (from Definition 1). Let $S_{2}=\left\{i^{\prime}: F_{i^{\prime}, 2}^{\prime} \geq\right.$ $2 c-i\}$ i.e., $S_{2}$ is the set of colors whose second facility for coloring $\mathrm{C}^{\prime}$ is at position $2 c-i$ or higher. Since $F_{i^{\prime}, 1}^{\prime}<2 c-i \forall i^{\prime},\left|S_{2}\right|=i+1$. Therefore $\left|S_{1}\right|+\left|S_{2}\right|>c$, i.e., $S_{1}$ and $S_{2}$ are not disjoint and there exists some $i^{\prime}$ such that $F_{i^{\prime}, 1}^{\prime} \geq F_{i, 1}$ and $F_{i^{\prime}, 2}^{\prime} \geq F_{i, 2} \Rightarrow L\left(F_{i^{\prime}, 1}^{\prime}, F_{i^{\prime}, 2}^{\prime}\right) \geq L(i+1,2 c-i)=L\left(F_{i, 1}, F_{i, 2}\right) \Rightarrow \alpha_{i^{\prime}, 1}^{\prime} \geq \alpha_{i, 1}$.

Case 2: $\ell=2$ : Let $d_{i}^{\prime}=d_{n+2-i} \forall i=1, \ldots, n+1$. Then, for the distance vector $\bar{d}^{\prime}=\left\langle d_{1}^{\prime}, \ldots, d_{n+1}^{\prime}\right\rangle$, the value of the objective function is $\alpha_{i, 1}$. This is because if the Voronoi length of the 2nd facility for color $i$ is the value of the objective function for coloring $C_{1}$, if we look at the mirror image such that the $\ell$-th facility from the left is now the $\ell$-th facility from the right, then the Voronoi length of the 1st facility for color $i$ is the value of the objective function for coloring $C_{1}$. So, as demonstrated in Case 1 , for the distance vector $\bar{d}^{\prime}$, for any new coloring $\mathrm{C}^{\prime}, \exists$ some $i^{\prime}$ such that $F_{i^{\prime}, 1}^{\prime} \geq F_{i, 1}$ and $F_{i^{\prime}, 2}^{\prime} \geq F_{i, 2}$. Hence for the distance vector $\bar{d}=\left\langle d_{1}, \ldots, d_{n+1}\right\rangle$, for any coloring $\mathrm{C}^{\prime}$, there exists some $i^{\prime}$ such that $F_{i^{\prime}, 1}^{\prime} \leq F_{i, 1}$ and $F_{i^{\prime}, 2}^{\prime} \leq F_{i, 2}$. Therefore, there exists some $i^{\prime}$ such that $F_{i^{\prime}, 1}^{\prime} \leq F_{i, 1}$ and $F_{i^{\prime}, 2}^{\prime} \leq F_{i, 2} \Rightarrow R\left(F_{i^{\prime}, 1}^{\prime}, F_{i^{\prime}, 2}^{\prime}\right) \geq R\left(F_{i, 1}, F_{i, 2}\right) \Rightarrow \alpha_{i^{\prime}, 2}^{\prime} \geq \alpha_{i, 2}$.

Hence, no coloring $\mathrm{C}^{\prime}$ can result in an objective function with value less than $\Delta\left(\mathrm{C}_{1}\right)$.

\section{$4 \quad n=k c, k>2$}

In this section, we extend the $\mathrm{C}_{1}$-coloring result of the previous section to the cases where $n=k c$ and $k>2$.

Theorem 2. For $n=k c$ and any integer $k>2, \mathrm{C}_{1}$ is an optimal coloring for the MinVor problem.

Proof. As the facilities are colored by the coloring $C_{1}$, let Voronoi length of the $j$-th facility of the color $\eta$ achieve maximum amongst all the facilities, i.e. $\Delta\left(C_{1}\right)=\alpha_{\eta, j}$. If $j=1$ or $k$, then we are looking at facilities at the left or right end among all the facilities of color $\eta$. Else, we are dealing with some intermediate facility of color $\eta$.

Consider first the case $j \notin\{1, k\}$. Let $S=\left\{F_{\eta, j-1}+1, F_{\eta, j-1}+2, \ldots, F_{\eta, j+1}-\right.$ $1\}$. We investigate if it is possible to achieve an objective function with value smaller than $\Delta\left(\mathrm{C}_{1}\right)$ by the alternate coloring scheme $\mathrm{C}^{\prime}$. There exists an $i$ such that for the coloring $\mathrm{C}^{\prime}$ there is at most one facility of color $i$ in $S$ because there are $c$ colors and $|S|=2 c-1$. Let there be one facility, say the $j_{1}$-th of color $i$ with a position in $S$ and $j_{\max }$ is the number of facilities of color $i$. Then for $1<j_{1}<j_{\max }, F_{i, j_{1}-1}^{\prime} \leq F_{\eta, j-1}<F_{i, j_{1}}^{\prime}<F_{\eta, j+1} \leq F_{i, j_{1}+1}^{\prime}$ which 
implies $\alpha_{i, j_{1}}^{\prime} \geq \alpha_{\eta, j}=\Delta\left(\mathrm{C}_{1}\right)$. If $j_{1}=1, F_{\eta, j-1}<F_{i, 1}^{\prime}<F_{\eta, j+1} \leq F_{i, 2}^{\prime}$ which implies $\alpha_{i, j_{1}}^{\prime}=L\left(F_{i, 1}^{\prime}, F_{i, 2}^{\prime}\right) \geq \alpha_{\eta, j}=\Delta\left(\mathrm{C}_{1}\right)$. If $j_{1}=j_{\max }, F_{i, j_{\max }-1}^{\prime} \leq F_{\eta, j-1}<$ $F_{i, j_{\max }}^{\prime}<F_{\eta, j+1}$ which implies $\alpha_{i, j_{1}}^{\prime}=R\left(F_{i, j_{\max }-1}^{\prime}, F_{i, j_{\max }}^{\prime}\right) \geq \alpha_{\eta, j}=\Delta\left(\mathrm{C}_{1}\right)$.

For $j=1$, the situation is similar to the Case 1 of Theorem 1 . We have $\Delta\left(\mathrm{C}_{1}\right)=\alpha_{\eta, 1}=L(\eta+1,2 c-\eta)$. We investigate if it is possible to achieve an objective function with value smaller than $\Delta\left(\mathrm{C}_{1}\right)$ by $\mathrm{C}^{\prime}$. Let $S_{1}=\left\{i^{\prime}: F_{i^{\prime}, 1}^{\prime} \geq\right.$ $\left.F_{\eta, 1}\right\}$. Since $F_{\eta, 1}=\eta+1,\left|S_{1}\right| \geq c-\eta$. Let $S_{2}=\left\{i^{\prime}: F_{i^{\prime}, 2}^{\prime} \geq F_{\eta, 2}\right\}$. Since $F_{\eta, 2}=2 c-\eta$ and $F_{i^{\prime}, 1}^{\prime}<F_{\eta, 2} \forall i^{\prime},\left|S_{2}\right| \geq \eta+1$. Therefore $\left|S_{1}\right|+\left|S_{2}\right|>c$, i.e., $S_{1}$ and $S_{2}$ are not disjoint and $\exists$ some $i^{\prime}$ such that $F_{i^{\prime}, 1}^{\prime} \geq F_{\eta, 1}$ and $F_{i^{\prime}, 2}^{\prime} \geq F_{\eta, 2} \Rightarrow$ $L\left(F_{i^{\prime}, 1}^{\prime}, F_{i^{\prime}, 2}^{\prime}\right) \geq L\left(F_{\eta, 1}, F_{\eta, 2}\right) \Rightarrow \alpha_{i^{\prime}, 1}^{\prime} \geq \alpha_{\eta, 1}$.

For $j=k$, we have $\Delta\left(\mathrm{C}_{1}\right)=\alpha_{\eta, k}=L(n-2 c+u, n-u+1)$ for some $u \in$ $\{1, \ldots, c\}$. Let $j_{i^{\prime}}$ be the number of facilities assigned to the color $i^{\prime}$ by the new coloring scheme $\mathrm{C}^{\prime}$ for which we investigate if it is possible to achieve an objective function with value smaller than $\Delta\left(C_{1}\right)$. Note that if $F_{i^{\prime}, j_{i^{\prime}}}^{\prime} \leq F_{\eta, k-1}$ for some $i^{\prime}$, then $R\left(F_{i^{\prime}, j_{i^{\prime}-1}}^{\prime}, F_{i^{\prime}, j_{i^{\prime}}}^{\prime}\right) \geq R\left(F_{\eta, k-1}, F_{\eta, k}\right)$ since $F_{i^{\prime}, j_{i^{\prime}}-1}^{\prime}<F_{i^{\prime}, j_{i^{\prime}}}^{\prime} \leq F_{\eta, k-1}<$ $F_{\eta, k}$. Hence we assume $F_{i^{\prime}, j_{i^{\prime}}}^{\prime}>F_{\eta, k-1} \forall i^{\prime}$. Let $S_{1}^{\prime}=\left\{i^{\prime}: F_{i^{\prime}, j_{i^{\prime}}}^{\prime} \leq F_{\eta, k}\right\}$. Since $F_{\eta, k}=n-u+1,\left|S_{1}^{\prime}\right| \geq c-u+1$. Let $S_{2}^{\prime}=\left\{i^{\prime}: F_{i^{\prime}, j_{i^{\prime}}-1}^{\prime} \leq F_{\eta, k-1}\right\}$. Since $F_{\eta, k-1}=n-2 c+u$ and $F_{i^{\prime}, j_{i^{\prime}}}^{\prime}>F_{\eta, k-1} \forall i^{\prime},\left|S_{2}^{\prime}\right| \geq u$. Therefore $\left|S_{1}^{\prime}\right|+\left|S_{2}^{\prime}\right|>c$, i.e., $S_{1}^{\prime}$ and $S_{2}^{\prime}$ are not disjoint and $\exists$ some $i^{\prime}$ such that $F_{i^{\prime}, j_{i^{\prime}}}^{\prime} \leq F_{\eta, k}$ and $F_{i^{\prime}, j_{i^{\prime}}-1}^{\prime} \leq F_{\eta, k-1} \Rightarrow R\left(F_{i^{\prime}, j_{i^{\prime}}-1}^{\prime}, F_{i^{\prime}, j_{i^{\prime}}}^{\prime}\right) \geq R\left(F_{\eta, k-1}, F_{\eta, k}\right) \Rightarrow \alpha_{i^{\prime}, j_{i^{\prime}}}^{\prime} \geq \alpha_{\eta, k}$.

\section{$5 \quad n=k c+m$}

In this section we consider the general case where $n$ is not a multiple of $c$. Note that $0<m<c$. In Section 5.1, we introduce a coloring $C_{2}$ where the facility of any color and its next to next neighbouring facilities of the same color have exactly $2 c-1$ facilities in between them. If the maximum Voronoi length among all the facilities in this coloring corresponds to an interior facility of some color, i.e, a facility which is neither the leftmost nor the rightmost of its color, then $\mathrm{C}_{2}$ is the optimal coloring. Otherwise, for a given $\Delta$, we define a coloring $\mathrm{C}_{\Delta}$ in Section 5.2. We denote the Voronoi length of the $j$-th facility of the $i$-th color for coloring $\mathrm{C}_{2}$ as $\beta_{i, j}$ and for coloring $\mathrm{C}_{\Delta}$ as $\gamma_{i, j}$. In Theorem 3 we show that there exists an optimal $\mathrm{C}_{\Delta}$ which can be obtained in $O\left(n^{2} \log n\right)$ time.

\subsection{Coloring $\mathrm{C}_{2}$}

Definition 2. (Coloring $C_{2}$ ): Let $S_{1}=0,1, \ldots, m-1, \bar{S}_{1}=m-1, m-$ $2, \ldots, 1,0, S_{2}=m, m+1, \ldots, c-1, \bar{S}_{2}=c-1, c-2, \ldots, m+1, m$. Consider $a$ coloring of the facilities from the left in the order $S_{1}, S_{2}, \bar{S}_{1}, \bar{S}_{2}, S_{1}, S_{2}, \bar{S}_{1}, \bar{S}_{2}, \ldots$ We define this assignment of colors as the coloring $\mathrm{C}_{2}$.

Lemma 1 If the value of the objective function for $\mathrm{C}_{2}$ is not equal to $\beta_{i, 1}$ or $\beta_{i, k}$ for some $i \in\{m, m+1, \ldots, c-1\}$, then $C_{2}$ is an optimal coloring.

Proof. The value of the objective function for $C_{2}$ can not be equal to $\beta_{i^{\prime}, 1}$ or $\beta_{i^{\prime}, k+1}$ for some $i^{\prime} \in\{0, \ldots, m-1\}$. By Definition $2, \forall i^{\prime} \in\{0,1, \ldots, m-1\}$ and $\forall i \in\{m, m+1, \ldots, c-1\}, P_{i^{\prime}, 1}=i^{\prime}+1<i+1=P_{i, 1}<P_{i^{\prime}, 2}=c+i^{\prime}+1<$ 
$c+i+1=P_{i, 2}$, which implies $\beta_{i^{\prime}, 1}=L\left(P_{i^{\prime}, 1}, P_{i^{\prime}, 2}\right)<L\left(P_{i, 1}, P_{i, 2}\right)=\beta_{i, 1}$. Similarly $P_{i^{\prime}, k}<P_{i, k-1}<P_{i^{\prime}, k+1}<P_{i, k}$ and hence $\beta_{i^{\prime}, k}=R\left(P_{i^{\prime}, k}, P_{i^{\prime}, k+1}\right)<$ $R\left(P_{i, k-1}, P_{i, k}\right)=\beta_{i, k}$.

Consider the case where the value of the objective function for $C_{2}$ is $\beta_{i, j}$ for some intermediate facility $j$ of any color $i$. We call $j$-th facility of a color $i$ as an intermediate facility when $2 \leq j \leq k-1$ for $i \in\{m, m+1, \ldots, c-1\}$ or when $2 \leq j \leq k$ for $i \in\{0,1, \ldots, m-1\})$. Then, by Definition $2, P_{i, j+1}-P_{i, j-1}=2 c$. Any alternate coloring scheme $C^{\prime}$ will have at least one color $i^{\prime}$ with at most one facility in $S=\left\{P_{i, j-1}+1, \ldots, P_{i, j+1}-1\right\}$. If there is one facility of $i^{\prime}$ in $S$, then its Voronoi length is at least $\beta_{i, j}$ and if there is no facility of $i^{\prime}$ in $S$, then the Voronoi length of a facility of color $i^{\prime}$ nearest to $S$ is greater that $\beta_{i, j}$. Hence the value of the objective function can not be reduced from $\beta_{i, j}$ by any different coloring scheme $\mathrm{C}^{\prime}$.

\subsection{Coloring $\mathrm{C}_{\Delta}$}

If the objective function for $\mathrm{C}_{2}$ returns the Voronoi length of the 1st or last facility of some color in $\{m, m+1, \ldots, c-1\}, C_{2}$ need not be optimal. For example if $n=18, c=5$ and the distance vector $d=(2,10,1,1,1,8,1,1,1,1,1,1,1,1,1,1,1,1,1)$, then $C_{2}$ returns an objective function 20.5 where as $O p t=19$ for the coloring scheme $0,1,2,3,4,4,3,2,1,0,3,0,1,2,4,2,1,0$. To handle such cases, let us consider a number $\Delta$ such that $\max \left(\max _{u=1, \ldots, n-2 c} M(u, u+2 c), \max _{u=1, \ldots, c} L(u, 2 c-u+\right.$ $\left.1), \max _{u=1, \ldots, c} R(n-2 c+u, n-u+1)\right) \leq \Delta \leq \max \left(M(1, n), \max _{u=1, \ldots, c} L(u, n-\right.$ $\left.u+1), \max _{u=1, \ldots, c} R(u, n-u+1)\right)$. The lower bound of $\Delta$ will be clear from Observation 1 and the upper bound is arrived considering the extreme cases where the 1st and 3rd facilities of a colour are at positions 1 and $n$ respectively, or the 1st and 2nd facilities of a colour are at positions $u$ and $n-u+1$ respectively for some $u \in\{1,2, \ldots, c\}$, or the last but one and last but one facilities of a colour are at positions $u$ and $n-u+1$ respectively for some $u \in\{1,2, \ldots, c\}$. We intend to define a Coloring $C_{\Delta}$ where the value of the objective function will be lesser than or equal to $\Delta$. If $\Delta$ is less than the optimum, clearly the coloring doesn't exist. There are $O\left(n^{2}\right)$ possible choices for $\Delta$ since the optimum can be of the form $L(a, b), M(a, b)$ or $R(a, b)$, where $1 \leq a<b \leq n$.

Observation 1 If $\max _{u=1, \ldots, n-2 c} M(u, u+2 c)>\Delta$ or $\max _{u=1, \ldots, c} L(u, 2 c-u+$ 1) $>\Delta$ or $\max _{u=1, \ldots, c} R(n-2 c+u, n-u+1)>\Delta$, then $\mathrm{C}_{\Delta}$ does not exist.

Proof. Proof omitted due to paucity of space.

After choosing a $\Delta$ at least as large as the maximum among $\max _{u=1, \ldots, n-2 c} M(u, u+2 c), \max _{u=1, \ldots, c} L(u, 2 c-u+1)$ and $\max _{u=1, \ldots, c} R(n-$ $2 c+u, n-u+1$ ), our scheme to obtain $\mathrm{C}_{\Delta}$ can be divided into two parts. The first part assigns $k$ facilities to each of the colors $m, \ldots, c-1$, ensuring that the Voronoi length of each of these $k(c-m)$ facilities is lesser than or equal to $\Delta$. The second part assigns the remaining colors $0, \ldots, m-1$ to the remaining $m(k+1)$ facilities, each of these colors being assigned to $k+1$ facilities. 
In the first part of the coloring scheme, we identify the facilities to be assigned the colors $m, \ldots, c-1$. Initially we define the positions of the first facilities of these colors as $P_{i, 1}=i+1 \forall i \in\{m, \ldots, c-1\} . P_{c-1,2}$ is such that $L\left(P_{c-1,1}, P_{c-1,2}\right) \leq \Delta<L\left(P_{c-1,1}, P_{c-1,2}+1\right)$, i.e., the farthest positon to ensure that $\gamma_{c-1,1} \leq \Delta$.

Observation $2 P_{c-1,2} \geq c+1$

Proof. $\Delta$ is chosen such that $\Delta>\max _{u=1, \ldots, c} L(u, 2 c-u+1) \geq L(c, c+1)$. As $P_{c-1,1}=c$ and $P_{c-1,2}$ is such that $L\left(P_{c-1,1}, P_{c-1,2}\right) \leq \Delta<L\left(P_{c-1,1}, P_{c-1,2}+1\right)$, the observation follows.

Now $\forall i=c-2, \ldots, m$, we define $P_{i, 2}$ such that $L\left(i+1, P_{i, 2}\right) \leq \Delta<$ $L\left(i+1, P_{i, 2}+1\right)$. If $k$ is odd and $P_{i, 2}>2 c-i+2 m$, we set $P_{i, 2}=2 c-i+2 m$. If $k$ is even and $P_{i, 2}>2 c-i+m$, we set $P_{i, 2}=2 c-i+m$. If $P_{i, 2} \leq P_{i+1,2}$, we backtrack by reducing $P_{i+1,2}$ and if necessary even earlier defined assignments of colors. Essentially we reduce $P_{i+1,2}$ by one, but if that conflicts with $P_{i+2,2}$, we reduce that by 1 and so on. Please note that the operation of backtracking will stop at $P_{c-1,2}$ or before because of the lower bound discussed in Observation 2. We observe, similar to the Observation 2, the following:

Observation $3 P_{i, 2} \geq 2 c-i$

To assign the $j$-th facility of each of these colors, $\forall j=3, \ldots, k-1$ :

1. If $j$ is odd, we define $P_{i, j}$, where the order of $i$ is $m, \ldots, c-1$, i.e, first $P_{m, j}$ is identified, then $P_{m+1, j}$ and so on. If $k$ is odd, $P_{i, j}=(j-1) c+i+1$. If $k$ is even, $P_{i, j}$ is such that $M\left(P_{i, j-2}, P_{i, j}\right) \leq \Delta<M\left(P_{i, j-2}, P_{i, j}+1\right)$. If $i>m$ and $P_{i, j} \leq P_{i-1, j}$, we backtrack starting with $P_{i-1, j}=P_{i, j}-1$. If $i=m$ and $P_{i, j} \leq P_{i, j-1}$, we backtrack starting with $P_{i, j-1}=P_{i, j}-1$. For even $k$ and $P_{i, j}>j c+i+m+1$, we set $P_{i, j}=j c+i+m+1$.

2. If $j$ is even, the order of $i$ is $c-1, \ldots, m$ while defining $P_{i, j}$, i.e, first $P_{c-1, j}$ is identified, then $P_{c-2, j}$ and so on. We ensure that $M\left(P_{i, j-2}, P_{i, j}\right) \leq \Delta<$ $M\left(P_{i, j-2}, P_{i, j}+1\right)$. If $i<c-1$ and $P_{i, j} \leq P_{i+1, j}$, we backtrack starting with $P_{i+1, j}=P_{i, j}-1$. If $i=c-1$ and $P_{i, j} \leq P_{i, j-1}$, we backtrack starting with $P_{i, j-1}=P_{i, j}-1$. If $k$ is odd and $P_{i, j}>j c-i+2 m$, we set $P_{i, j}=j c-i+2 m$. If $k$ is even and $P_{i, j}>j c-i+m$, we set $P_{i, j}=j c-i+m$.

Observation $4 P_{i, j+1}-P_{i, j-1} \geq 2 c \forall j=2,3, \ldots, k-1$.

Proof. $\Delta$ is chosen such that $\Delta \geq \max _{u=1, \ldots, n-2 c} M(u, u+2 c)$. If $P_{i, j+1}-$ $P_{i, j-1}<2 c$ for any $j \in\{2,3, \ldots, k-1\}$, then $\gamma_{i, j}=M\left(P_{i, j-1}, P_{i, j+1}\right)<$ $M(u, u+2 c)$ for some $u$.

Observation $5 P_{i, j} \geq(j-1) c+i+1$ for odd $j$ and $P_{i, j} \geq j c-i$ for even $j$.

Proof. $P_{i, 1}=i+1$ and by Observation 4 for odd $j, P_{i, j}-P_{i, 1} \geq \frac{j-1}{2} 2 c \Rightarrow P_{i, j} \geq$ $(j-1) c+i+1$. By Observations 2 and $3, P_{i, 2} \geq 2 c-i$ for all $i \in\{m, m+$ $1, \ldots, c-1\}$. Therefore for even $j, P_{i, j}-P_{i, 2} \geq \frac{j-2}{2} 2 c \Rightarrow P_{i, j} \geq j c-i$. 
Now we define $P_{i, k}$, i.e., the last position for the colors $i=m, \ldots, c-1$. If $k$ is odd, we define $P_{i, k}=n-c-m+i+1 \forall i=m, \ldots, c-1$. If $k$ is even, we define $P_{i, k}=n-i \forall i=m, \ldots, c-1$. Irrespective of whether $k$ is odd or even, if $M\left(P_{i, k-2}, P_{i, k}\right)>\Delta$ for any $i \in\{m, \ldots, c-1\}$ or $R\left(P_{i, k-1}, P_{i, k}\right)>\Delta$ for any $i \in\{m, \ldots, c-1\}$, then $C_{\Delta}$ does not exist for the given $\Delta$. Otherwise we proceed to the second part of the coloring scheme.

In the second part of the scheme, we assign facilties to the colors $0, \ldots, m-1$. We have remaining $m(k+1)$ facilities to be colored $0, \ldots, m-1$. We color them using Coloring $C_{1}$ for $m$ colors. It is easy to note that,

Observation 6 For $i=0,1, \ldots, m-1$, we have $P_{i, 1}=i+1, P_{i, k+1}=n-i$ for odd $k$ and $P_{i, k+1}=n-m+i+1$ for even $k$.

\subsection{Optimality of $\mathrm{C}_{\Delta}$}

In this section we prove that an optimal coloring $C_{\Delta}$ and the corresponding $\Delta=O p t$ can be identified in $O\left(n^{2} \log n\right)$ time. Proof of Lemma 4 is omitted because of paucity of space.

Lemma 2 For all $i^{\prime} \in\{0, \ldots, m-1\}$

$$
\begin{aligned}
& \text { 1. } \gamma_{i^{\prime}, 1} \leq L(u, 2 c-u+1) \text { for some } u \in\{1, \ldots, c\} \\
& \text { 2. } \gamma_{i^{\prime}, k+1} \leq R(n-2 c+u, n-u+1) \text { for some } u \in\{1, \ldots, c\} \\
& \text { 3. } P_{i^{\prime}, j^{\prime}+1}-P_{i^{\prime}, j^{\prime}-1} \leq 2 c \forall j^{\prime} \in\{2, \ldots, k\} \text {. }
\end{aligned}
$$

Proof. If $2 c-i^{\prime}<P_{i^{\prime}, 2} \leq 2 c$ for some $i^{\prime} \in S_{1}=\{0, \ldots, m-1\}$, then to its left there are at least $2 c-i^{\prime}$ facilities of which the first $c$ are the first facilities of each color (by the description of $\mathrm{C}_{\Delta}$ and Observation 6). Moreover, there are $m-i^{\prime}-1$ more facilities with colors in $S_{1}$ with position less than $P_{i^{\prime}, 2}$. So in $\left\{c+1, \ldots, P_{i^{\prime}, 2}-1\right\}$ there are atleast $2 c-i^{\prime}-\left(m-i^{\prime}-1\right)=c-m+1$ facilities with colors in $S_{2}=\{m, \ldots, c-1\}$, which implies $P_{i^{\prime \prime}, 3}<P_{i^{\prime}, 2}<2 c$ for some $i^{\prime \prime} \in S_{2}$. Then $P_{i^{\prime \prime}, 3}-P_{i^{\prime \prime}, 1}<2 c$ violating Observation 4 . If $P_{i^{\prime}, 2}>2 c$, then there are at most $2 m-1$ of the facilities with position less than $P_{i^{\prime}, 2}$ are from $S_{1}$. Therefore there are at least $2 c-(2 m-1)=2(c-m)+1$ facilities assigned colors from $m, m+1, \ldots, c-1$ with positions in $\{1,2, \ldots, c\}$, i.e., there is a color $i \in\{m, m+1, \ldots, c-1\}$ such that $P_{i, 3} \leq 2 c$. But $P_{i, 1}>m$ for all $i \in\{m, m+1, \ldots, c-1\}$ and hence $P_{i, 3}-P_{i, 1}<2 c$ violating Observation 4 . So $P_{i^{\prime}, 2} \leq 2 c-i^{\prime}$ for all $i^{\prime} \in S_{1}$ and hence $\gamma_{i^{\prime}, 1} \leq L(u, 2 c-u+1)$ for some $u \in\{1, \ldots, c\}$.

Similarly $\gamma_{i^{\prime}, k+1} \leq R(n-2 c+u, n-u+1)$ for some $u \in\{1, \ldots, c\}$.

Suppose $\exists i^{\prime} \in S_{1}$ such that $P_{i^{\prime}, j^{\prime}+1}-P_{i^{\prime}, j^{\prime}-1}>2 c$. Let $T=\left\{P_{i^{\prime}, j^{\prime}-1}+\right.$ $\left.1, \ldots, P_{i^{\prime}, j^{\prime}+1}-1\right\}$ and $|T|=2 c-1+u$ where $u>0$. Since the colors in $S_{1}$ form an $C_{1}$ of $m$ colors for the $m(k+1)$ facilities they are assigned to, $\forall i_{1} \in S_{1}-\left\{i^{\prime}\right\}$ there are exactly two facilities in $T$. Hence there are $2 c-$ $1+u-(2 m-1)=2(c-m)+u$ facilities in $T$ with colors in $S_{2}$. Let colors $i_{1}, \ldots, i_{u} \in S_{2}$ have 3 facilities each in $T$. If the leftmost facility in $T$ of any color $i_{a} \in\left\{i_{1}, \ldots, i_{u}\right\}$ is positioned at $P_{i_{a}, j_{a}}>P_{i_{1}, j_{1}-1}+u$, then $P_{i_{a}, j_{a}+2}-P_{i_{a}, j_{a}}<$ 
$2 c$, violating Observation 4. Similarly, the rightmost facility in $T$ of any color $i_{a} \in\left\{i_{1}, \ldots, i_{u}\right\}$ can not positioned at $P_{i_{a}, j_{a}+2}<P_{i_{1}, j_{1}+1}-u$. Therefore, positions $P_{i_{1}, j_{1}-1}+1, \ldots, P_{i_{1}, j_{1}-1}+u$ are assigned to colors from $\left\{i_{1}, \ldots, i_{u}\right\}$ and positions $P_{i_{1}, j_{1}+1}-u, \ldots, P_{i_{1}, j_{1}+1}-1$ are assigned to colors from $\left\{i_{1}, \ldots, i_{u}\right\}$. Let $P_{i_{a}, j_{a}}=P_{i_{1}, j_{1}-1}+1$. If $P_{i_{a}, j_{a}+2}=P_{i_{1}, j_{1}+1}-u$, then Theorem $P_{i_{a}, j_{a}+2}-P_{i_{a}, j_{a}}=$ $2 c-1<2 c$, violating Observation 4. If $P_{i_{a}, j_{a}+2}>P_{i_{1}, j_{1}+1}-u$, then there exists an $i_{b}$ such that $P_{i_{b}, j_{b}+2}=P_{i_{1}, j_{1}+1}-u$ and $P_{i_{b}, j_{b}}>P_{i_{1}, j_{1}-1}+1$. Hence $P_{i_{b}, j_{b}+2}-P_{i_{b}, j_{b}}<\left(P_{i_{1}, j_{1}+1}-u\right)-\left(P_{i_{1}, j_{1}-1}+1\right)=2 c-1<2 c$, again violating Observation 4.

Lemma 3 If $\mathrm{C}_{\Delta}$ exists and $\gamma_{i, j}$ is the Voronoi length of the $j$-th facility of color $i$, then $\forall i$ and $\forall j, \gamma_{i, j} \leq \Delta$.

Proof. By the definition of $\mathrm{C}_{\Delta}$, if it exists, $\gamma_{i, j} \leq \Delta \forall i \in\{m, \ldots, c-1\}$, $\forall j \in\{1, \ldots, k\}$.

From Lemma 2 we have for all $i^{\prime} \in\{0, \ldots, m-1\}$

1. $\gamma_{i^{\prime}, 1} \leq L(u, 2 c-u+1)$ for some $u \in\{1, \ldots, c\}$

2. $\gamma_{i^{\prime}, k+1} \leq R(n-2 c+u, n-u+1)$ for some $u \in\{1, \ldots, c\}$

3. $P_{i^{\prime}, j^{\prime}+1}-P_{i^{\prime}, j^{\prime}-1} \leq 2 c \forall j^{\prime} \in\{2, \ldots, k\}$, i.e., $\forall j \in\{2, \ldots, k\}, \gamma_{i^{\prime}, j} \leq$ $M(u, u+2 c)$ for some $u \in\{1, \ldots, n-2 c\}$

Our coloring scheme for $\mathrm{C}_{\Delta}$ chooses a $\Delta$ at least as large as the maximum among $\max _{u=1, \ldots, n-2 c} M(u, u+2 c), \max _{u=1, \ldots, c} L(u, 2 c-u+1)$ and $\max _{u=1, \ldots, c} R(n-2 c+u, n-u+1)$. Therefore $\gamma_{i^{\prime}, j} \leq \Delta \forall i^{\prime} \in\{0, \ldots, m-1\}$, $\forall j \in\{1, \ldots, k+1\}$.

Lemma 4 If $\mathrm{C}_{\mathrm{a}}$ is an optimal coloring where the colors of the facilities $1,2, \ldots, c$ are not distinct or the colors of the facilities $n-c+1, n-c+2, \ldots, n$ are not distinct, there exists another optimal coloring $\mathrm{C}_{\mathrm{b}}$ where the colors of the facilities $1,2, \ldots, c$ are distinct and the colors of the facilities $n-c+1, n-c+2, \ldots, n$ are distinct.

Lemma 5 If $O p t \leq \Delta$, there exists a $\mathrm{C}_{\Delta}$ where the value of the objective function is $\Delta$.

Proof. Suppose, if possible, there exists some $\Delta \geq O p t$ and the coloring scheme announced that $\mathrm{C}_{\Delta}$ does not exist for such $\Delta$. This announcement is made if, for some $i \in\{m, \ldots, c-1\}$, at least one of the following occurs:

1. $M\left(P_{i, k-2}, P_{i, k}\right)>\Delta$

2. $R\left(P_{i, k-1}, P_{i, k}\right)>\Delta$

If $M\left(P_{i, k-2}, P_{i, k}\right)>\Delta$ and there would exist a coloring $\mathrm{C}^{\prime}$ where the $j$-th facility for color $\eta$ is at position $P_{\eta, j}^{\prime}$, has Voronoi length $\gamma_{\eta, j}^{\prime}$, $\max _{i=0, \ldots, c-1} \max _{j=1, \ldots, j_{\max }} \gamma_{\eta, j}^{\prime} \leq \Delta$ and at least one of the following is true:

1. $P_{i, k-2}^{\prime}>P_{i, k-2}$

2. $P_{i, k}^{\prime}<P_{i, k}$ 
As is obvious from the definition of $\mathrm{C}_{\Delta}, P_{i, k-2}$ could not be increased without increasing $\gamma_{i, k-1}$ or $\gamma_{\eta, u}$ for some $\eta$ and some $u \leq k-1$. So if $P_{i, 1}^{\prime}=P_{i, 1}=i+1$, then $P_{i, k-2}^{\prime}>P_{i, k-2}$ would imply that the value of the objective function for $\mathrm{C}^{\prime}$ is greater than $\Delta$. Lemma 4 suggests for every optimal coloring we can have a recoloring such that $P_{i, 1}^{\prime}=P_{i, 1}=i+1 \forall i \in\{0,1, \ldots, c-1\} . P_{i, k}^{\prime}<P_{i, k}$ can not be achieved because by Lemma 4 there exists an optimal coloring such that the colors of the facilities $n-c+1, n-c+2, \ldots, n$ are distinct and if $P_{i, 1}^{\prime}=P_{i, 1}=i+1 \forall i \in\{0,1, \ldots, c-1\}$, the maximum Voronoi length will only increase if $P_{i, k}^{\prime}<P_{i, k}$ since it would violate the $\mathrm{C}_{1}$ coloring for the colors $m, m+1, \ldots, c-1$.

If $R\left(P_{i, k-1}, P_{i, k}\right)>\Delta$ and there would exist a coloring $\mathrm{C}^{\prime}$ where the $j$-th facility for color $\eta$ is at position $P_{\eta, j}^{\prime}$, has Voronoi length $\gamma_{\eta, j}^{\prime}$, $\max _{i=0, \ldots, c-1} \max _{j=1, \ldots, j_{\max }} \gamma_{\eta, j}^{\prime} \leq \Delta$ and at least one of the following is true:

1. $P_{i, k-1}^{\prime}>P_{i, k-1}$

2. $P_{i, k}^{\prime}>P_{i, k}$

But, as explained above while considering the case $P_{i, k-2}^{\prime}>P_{i, k-2}$, we can not have $P_{i, k-1}^{\prime}>P_{i, k-1}$ if $P_{i, 1}^{\prime}=P_{i, 1}=i+1 \forall i \in\{0,1, \ldots, c-1\} . P_{i, k}^{\prime}>P_{i, k}$ would violate the $C_{1}$ coloring for the colors $m, m+1, \ldots, c-1$ as it would for $P_{i, k}^{\prime}<P_{i, k}$.

Theorem 3. For $n=k c+m, 0<m<c$, an optimal coloring scheme can be obtained in $O\left(n^{2} \log n\right)$ time.

Proof. From Lemma 5 it is clear that there exists a $\Delta$ such that $C_{\Delta}$ is optimal. A candidate for $\Delta$ is any of the following:

1. $L(a, b)$ where $1 \leq a<b \leq n$

2. $M(a, b)$ where $1 \leq a<b \leq n$

3. $R(a, b)$ where $1 \leq a<b \leq n$

There are $O\left(n^{2}\right)$ candidates for $\Delta$, which can be sorted in ascending order in $O\left(n^{2} \log n\right)$ time. For a given $\Delta$, we can identify $\mathrm{C}_{\Delta}$ in $O(c n)$ time as follows. We have a list of $n$ cumulative distances $D_{j}=\Sigma_{i=1}^{j} d_{i} \forall j=1,2, \ldots, n$. One can calculate $L(a, b)=\frac{D_{a}+D_{b}}{2}, M(a, b)=\frac{D_{b}-D_{a}}{2}$ and $R(a, b)=d_{n+1}+D_{n}-\frac{D_{a}+D_{b}}{2}$ for any pair of $a$ and $b$ in constant time. For odd $k, P_{i, j}$ is fixed for odd $j$ and, for even $j, j c-i \leq P_{i, j} \leq j c-i+2 m$. For even $k, j c+i \leq P_{i, j} \leq j c+i+m+1$ for odd $j$ and $j c-i \leq P_{i, j} \leq j c-i+m$ for even $j$. So, for identifying $P_{i, j}$ for $i \in\{m, m+1, \ldots, c-1\}$ using the rule $M\left(P_{i, j-2}, P_{i, j}\right) \leq \Delta \leq M\left(P_{i, j-2}, P_{i, j}+1\right)$ for $j>2$ or $L\left(P_{i, 1}, P_{i, 2}\right) \leq \Delta \leq L\left(P_{i, 1}, P_{i, 2}+1\right)$, we need $O(m)$ time for each $i, j$ and hence $O(c n)$ time for all the facilities as $m<c$. For backtracking we need:

1. At most $c-i$ time for odd $k$ and even $j$ for each $i \in\{m, m+1, \ldots, c-1\}$

2. No backtracking for odd $k$ and odd $j$

3. At most $c-i$ time for even $k$ and even $j$ for each $i \in\{m, m+1, \ldots, c-1\}$

4. At most $m$ time for even $k$ and odd $j$ for each $i \in\{m, m+1, \ldots, c-1\}$

So for backtracking we need $O(c n)$ time and altogether we need $O(c n)$ time for each $\Delta$. Using binary search over the sorted list of $O\left(n^{2}\right)$ candidates for $\Delta$, we obtain the optimum coloring by trying atmost $O(\log n)$ candidates. Hence the total time complexity is $O(c n \log n)+O\left(n^{2} \log n\right)$, i.e., $O\left(n^{2} \log n\right)$. 


\section{Conclusions}

With the objective of coloring the available facilities to a population of users distributed uniformly in a line segment $R$ such that the load of the different facilities are distributed as equitably as possible (MinVor problem) we obtained some interesting results. We observed that when $n=k c, \mathrm{C}_{1}$ offers us an optimal coloring for any distance vector, while for $n=k c+m$ facilities with $c$ colors for the MinVor problem, we have $C_{2}$ is the optimal coloring in some special cases and otherwise $\mathrm{C}_{\Delta}$ is the optimal coloring with $\Delta$ being the optimal value which can be obtained in $O\left(n^{2} \log n\right)$ time.

If $\Delta<\max _{u=1, \ldots, n-2 c} M(u, u+2 c)=M\left(u_{1}, u_{1}+2 c\right)$, there must be a color $i$ with only one facility in $\left\{u_{1}+1, \ldots, u_{1}+2 c-1\right\}$. The Voronoi length of that facility of color $i$ is clearly atleast $M\left(u_{1}, u_{1}+2 c\right)>\Delta$.

Let $\Delta<\max _{u=1, \ldots, c} L(u, 2 c-u+1)=L\left(u_{2}, 2 c-u_{2}+1\right)$. If the first facility of color $i$ is at position $u_{2}$ or higher, we say that it follows Property 1 . If the 2nd facility of color $i$ is at position $\left(2 c-u_{2}+1\right)$ or higher, we say that it follows Property 2. Clearly at least $c-u_{2}+1$ colors follow Property 1 and $u_{2}$ colors follow Property 2. So, there is at least one color, say $i_{1}$, whose 1 st facility is at position $u_{2}$ or higher and 2 nd facility at $2 c-u_{2}+1$ or higher. Clearly $L\left(P_{i_{1}, 1}, P_{i_{1}, 2}\right) \geq L\left(u_{2}, 2 c-u_{2}+1\right)>\Delta$.

Similarly if $\Delta<\max _{u=1, \ldots, c} R(n-2 c+u, n-u+1)=R\left(n-2 c+u_{3}, n-\right.$ $\left.u_{3}+1\right)$, there is at least one color, say $i_{2}$, whose last facility is at position $n-u_{3}+1$ or lower and the 2 nd last facility at $n-2 c+u_{3}$ or lower, which implies $R\left(P_{i_{2}, j-1}, P_{i_{2}, j}\right) \geq R\left(n-2 c+u_{3}, n-u_{3}+1\right)>\Delta$, where $j$ is the total number of facilities of color $i_{2}$.

\section{References}

1. Stefan Funke, Alexander Kesselman, Fabian Kuhn, Zvi Lotker, Michael Segal: Improved approximation algorithms for connected sensor cover. Wireless Networks 13(2): 153-164 (2007)

2. Ying Lin, Jun Zhang, Henry Shu-Hung Chung, Wai-Hung Ip, Yun Li, Yu-hui Shi: An Ant Colony Optimization Approach for Maximizing the Lifetime of Heterogeneous Wireless Sensor Networks. IEEE Transactions on Systems, Man, and Cybernetics, Part C 42(3): 408-420 (2012)

3. Brent N. Clark, Charles J. Colbourn, David S. Johnson: Unit disk graphs. Discrete Mathematics 86(1-3): 165-177 (1990)

4. Peng-Jun Wan, Khaled M. Alzoubi, Ophir Frieder: Distributed Construction of Connected Dominating Set in Wireless Ad Hoc Networks. MONET 9(2): 141-149 (2004)

5. Stefan Funke, Alexander Kesselman, Ulrich Meyer, Michael Segal: A simple improved distributed algorithm for minimum CDS in unit disk graphs. TOSN 2(3): 444-453 (2006) 